\title{
Astrophysical Implications of Strong-Field QED
}

\author{
Jeremy S. Heyl \\ University of British Columbia - Vancouver - Canada
}




\section{Astrophysical Implications of Strong-Field QED}

Jeremy S. Heyl

25/26 April 2012

Students: Ramandeep Gill, Kelsey Hoffman, Dan Mazur, Alain Prat, Anand Thirumalai

Others: Dong Lai (Cornell), Nir Shaviv (Jerusalem), Kaya Mori (CITA), Yoram Lithwick (Northwestern)

http://bit.ly/heylqed 


\section{Outline}

Objects

Neutron Stars

White Dwarfs

Black Holes

Processes

Vacuum Breakdown

Vacuum Polarization

Axions and Plasmas

Photon Splitting

Magnetars

Observations 


\section{Stars}

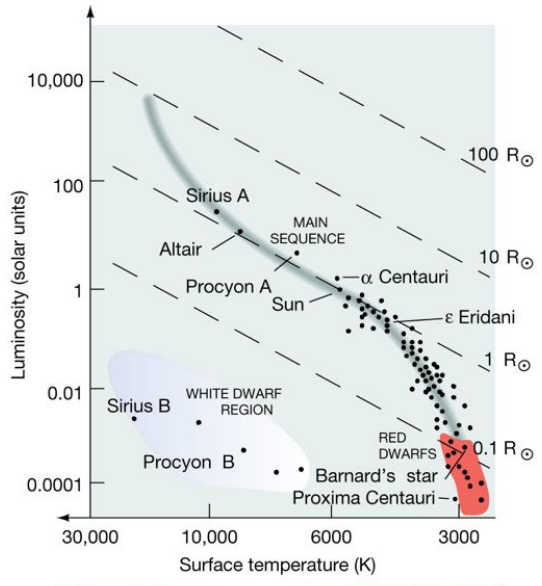

Nearby Stars

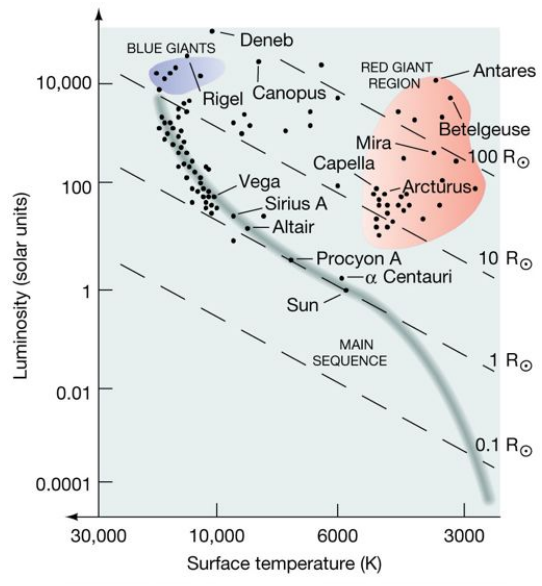

Bright Stars 


\section{How big are compact objects?}

White Dwarfs

Gravity yields: $P_{0} \sim \frac{G M^{2}}{R^{4}}$

Relativistic degenerate electrons

$$
P_{0} \sim \frac{m_{e} c^{2}}{\lambda_{e}^{3}}, M=\frac{m_{p}}{\lambda_{e}^{3}} R^{3}
$$

Solving yields

$$
\begin{gathered}
R=\lambda_{e} \frac{m_{P}}{m_{p}}, M=\frac{m_{P}^{3}}{m_{p}^{2}} \\
R \approx 10,000 \mathrm{~km}, M \approx 1.4 \mathrm{M}_{\odot}
\end{gathered}
$$

Neutron Stars

Gravity yields: $P_{0} \sim \frac{G M^{2}}{R^{4}}$ Relativistic degenerate neutrons

$$
P_{0} \sim \frac{m_{n} c^{2}}{\lambda_{n}^{3}}, M=\frac{m_{n}}{\lambda_{e}^{3}} R^{3}
$$

Solving yields

$$
\begin{gathered}
R=\lambda_{n} \frac{m_{P}}{m_{n}}, M=\frac{m_{P}^{3}}{m_{n}^{2}} \\
R \approx 17 \mathrm{~km}, M \approx 1.4 \mathrm{M}_{\odot}
\end{gathered}
$$




\section{What is a neutron star?}

- The remnant of the explosion of massive star.

- A giant atomic nucleus.

- An accurate clock ( $\mu \mathrm{s})$

- A giant magnet

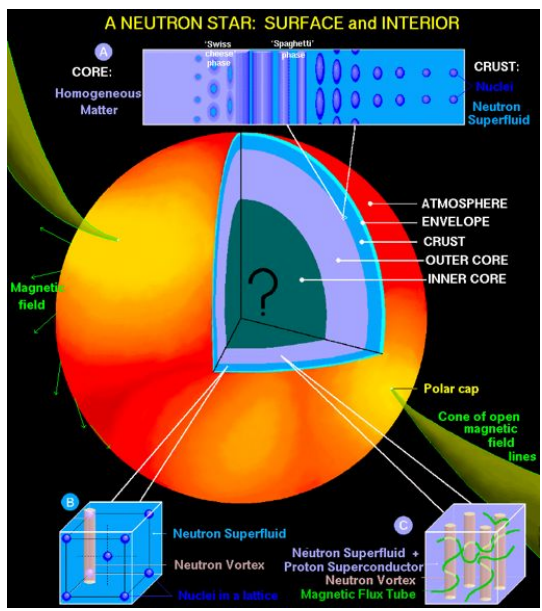

Dany Page 


\section{How strong can their fields be?}

Let's calculate the expected magnetic field of a neutron star.

- Let's assume that the core of the star has a magnetic field and mass similar to the Sun's.

- A neutron star has a radius of $10 \mathrm{~km}$.

- Flux freezing: $\Phi \propto B R^{2}$.

- $B \sim 50 \mathrm{G}(70000)^{2} \sim$ $10^{11-12} \mathrm{G}$.

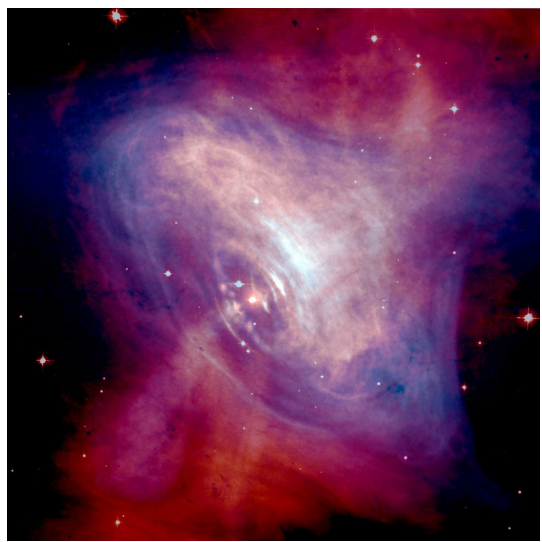




\section{Neutron Stars}

- The first neutron stars to be identified were radio pulsars.

- Over 2,000 are now known.

- Lots of flavours - not even including the accretors.

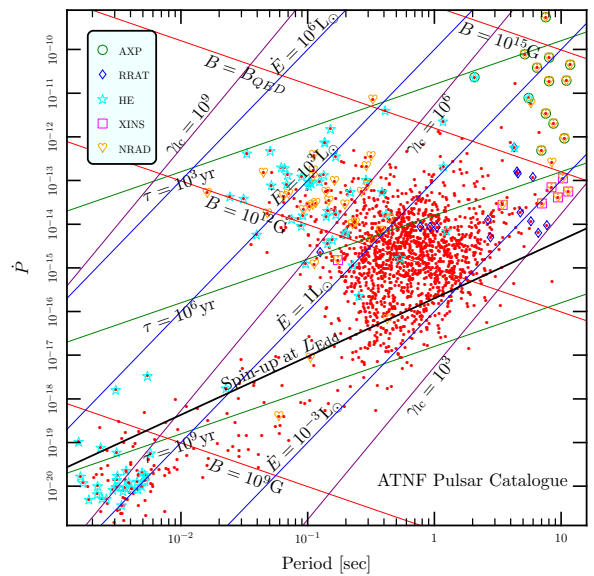




\section{Phenomenology of Neutron Stars}

The figure on the previous page has tons of lines giving various physical quantities based on the values of $P$ and $\dot{P}$. The basic premise behind these lines is that the spin-down of the star is due to magnetic dipole radiation. In particular we have

$$
\dot{E}=I \Omega \dot{\Omega}=-\frac{B_{p}^{2} R^{6} \Omega^{4} \sin ^{2} \alpha}{6 c^{3}}
$$

where $\Omega$ is the angular frequency of the pulsar, $I \approx 10^{45} \mathrm{gcm}^{2}$ is the moment of inertia, $B_{p}$ is the strength of the dipolar component of the magnetic field at the surface of radius $R \approx 10 \mathrm{~km}$ and $\alpha$ is the angle between the spin axis and the dipole axis. This equation yields an estimate for the energy loss

$$
\dot{E}=4 \times 10^{46} \mathrm{erg} / \mathrm{s}\left(\frac{P}{1 \mathrm{sec}}\right)^{-3} \dot{P}=10^{13} \mathrm{~L}_{\odot} P_{1}^{-3} \dot{P},,
$$

[ surface dipolar magnetic field and age

$$
B_{p}=2.5 \times 10^{19} \mathrm{G} \sqrt{\dot{P} P_{1}}, \tau=0.5 P \dot{P}^{-1}
$$

If one assumes that the electric fields induced by the rotating magnetic field are not shorted out, then we can estimate the typical EMF between different parts of the light cylinder $(c / \Omega)$ and the value of $\gamma$ acquired by an electron through the EMF. We have (GJ)

$$
\Phi_{\mathrm{GJ}}=\frac{B_{p} \Omega}{3 c} \frac{R^{5}}{r^{3}} P_{2}(\cos \theta) \text {. }
$$

If we take the Legendre polynomial to be unity this yields $\gamma \approx 10^{6} \dot{P}^{1 / 2} P_{1}^{-7 / 2}$. The final line assumes a model in which millisecond pulsars are produced when a neutron star accretes material from a companion. This both spins up the neutron star and weakens its magnetic field. If we assume that radiation pressure balances gravity in the accretion flow, we have

$$
L=L_{\mathrm{Edd}}=\frac{4 \pi c G M m_{p}}{\sigma_{T}}=1.3 \times 10^{38} \frac{\mathrm{M}}{\mathrm{M}_{\odot}} \mathrm{erg} / \mathrm{s} .
$$

and $L=\dot{M} G M / R$. Furthermore, we assume that the accretion flow surrounding the star is disrupted at the Alfven radius where magnetic pressure balances ram pressure

$$
r_{A}=\left(\frac{B_{p}^{4} R^{12}}{2 G M \dot{M}}\right)^{1 / 7}=\left(\frac{B_{p}^{4} R^{10} G M}{2 L^{2}}\right)^{1 / 7} .
$$

Finallly, we assume that the spin of the star at a given time equals the Keplerian angular velocity at $r_{A}$, $\sqrt{G M / r_{A}^{3}}$, yielding $\frac{L}{L_{\mathrm{Edd}}}=5 \times 10^{15} \dot{P} P_{1}^{-4 / 3}$. Note that this relates the accretion luminosity to the spin and spin-down rate right after the accretion ceases - it is spin-up line. 


\section{What is a white dwarf?}

- The remnant of the effluence of a sun-like star.

- A giant diamond

- An accurate clock (Gyr)

- A giant magnet 


\section{How strong can their fields be?}

Let's calculate the expected magnetic field of a white dwarf.

- The magnetic field of the Sun is about 50 Gauss.

- A white dwarf is the radius of the Earth.

- Flux freezing: $\Phi \propto B R^{2}$.

- $B \sim 50 \mathrm{G}(109)^{2} \sim$ $500 \mathrm{kG}=50 \mathrm{~T}$.

- Magnetic Ap stars have $1 \mathrm{kG}$ fields and are bigger than the Sun.

- $B \sim 100 \mathrm{MG}=10^{4} \mathrm{~T}$.

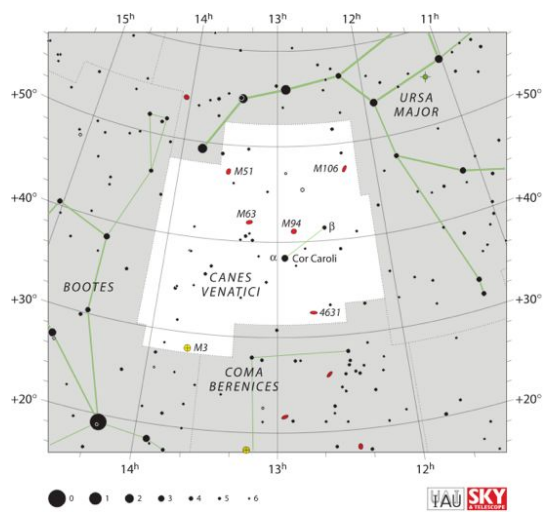




\section{White Dwarf Spectra}

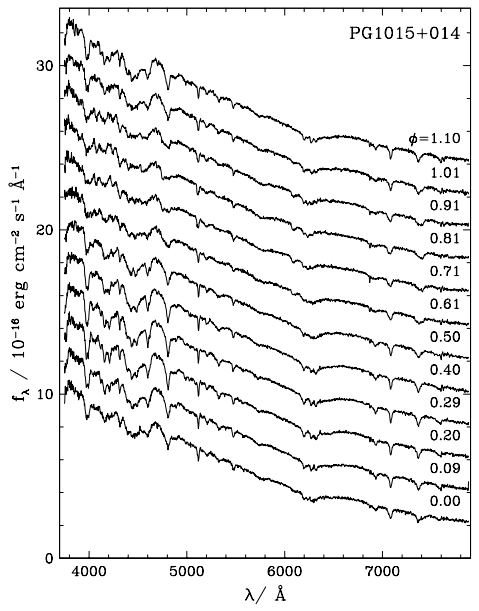

PG1015+014 : Left (Euchner et al. 2006), Below (Keck;Heyl)

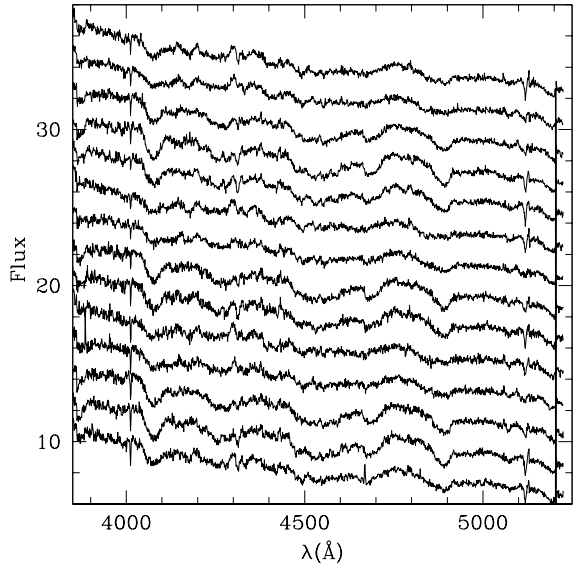




\section{What is a black hole?}

- The remnant of the explosion of a really massive star.

- A giant nothing

- Stops clocks

- No magnet allowed

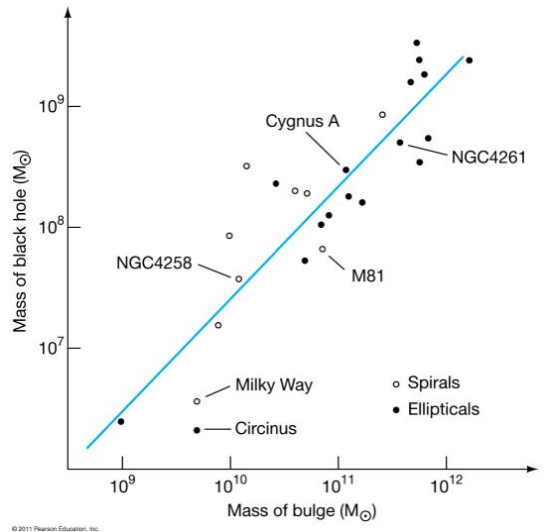




\section{Vacuum Breakdown in an External Field}

$$
\mathcal{L}_{\text {eff }}=\frac{\hbar}{8 \pi^{2}} B_{k}^{2} \int_{0}^{\infty} \frac{d \zeta}{\zeta} e^{-i \zeta}\left[\frac{a b}{B_{k}^{2}} \operatorname{coth}\left(\zeta \frac{a}{B_{k}}\right) \cot \left(\zeta \frac{b}{B_{k}}\right)-\mathrm{CT}\right]
$$

where

$$
\begin{gathered}
(b-i a)^{2}=(\mathbf{B}-i \mathbf{E})^{2}=|\mathbf{B}|^{2}-|\mathbf{E}|^{2}-2 i \mathbf{E} \cdot \mathbf{B} \\
{\left[2(b-i a)^{2}\right]=F^{\mu \nu} F_{\mu \nu}+i \epsilon_{\mu \nu \alpha \beta} F^{\mu \nu} F^{\alpha \beta} \equiv I+i J}
\end{gathered}
$$

and

$$
\mathrm{CT}=\frac{1}{\zeta^{2}}+\frac{1}{3} \frac{a^{2}-b^{2}}{B_{k}^{2}}\left(a^{2}-b^{2}\right)
$$




\section{Pair Production Rate}

The pair production rate is related to the imaginary part of

$$
\mathcal{L}_{\text {eff }}=\frac{\hbar}{8 \pi^{2}} B_{k}^{2} \int_{0}^{\infty} \frac{d \zeta}{\zeta} e^{-i \zeta}\left[\frac{a b}{B_{k}^{2}} \operatorname{coth}\left(\zeta \frac{a}{B_{k}}\right) \cot \left(\zeta \frac{b}{B_{k}}\right)-\mathrm{CT}\right] .
$$

The imaginary part of the integrand is even so we can extend the integration to the real line and complete with a semicircle.

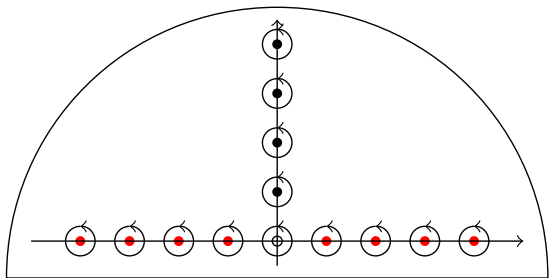




\section{Vacuum Breakdown}

- In general,

$$
w=\frac{1}{\pi}\left(\frac{\hbar}{m c}\right)^{-3}\left(\frac{\hbar}{m c^{2}}\right)^{-1} \frac{a b}{B_{k}^{2}} \sum_{n=1}^{\infty} \frac{1}{n} \operatorname{coth}\left(\frac{n \pi b}{a}\right) \exp \left(-n \pi \frac{B_{k}}{|a|}\right)
$$

- For weak magnetic fields $(a \gg b)$,

$$
w \approx \frac{1}{\pi^{2}}\left(\frac{\hbar}{m c}\right)^{-3}\left(\frac{\hbar}{m c^{2}}\right)^{-1} \frac{a^{2}}{B_{k}^{2}} \operatorname{dilog}\left[1-\exp \left(-\pi B_{k} /|a|\right)\right] .
$$

- For strong magnetic fields $(b \gg a)$,

$$
w \approx-\frac{1}{\pi}\left(\frac{\hbar}{m c}\right)^{-3}\left(\frac{\hbar}{m c^{2}}\right)^{-1} \frac{a b}{B_{k}^{2}} \log \left[1-\exp \left(-\pi B_{k} /|a|\right)\right] .
$$




\section{Vacuum Breakdown near Rotating Black Holes}

In a vacuum spacetime, a linear combination of Killing vectors yields a solution for the electromagnetic vector potential also in vacua.

$$
A_{\mu}=\frac{1}{2} B_{0}\left(\psi_{\mu}+\frac{2 J}{M} \eta_{\mu}\right)-\frac{Q}{2 M} \eta_{\mu}
$$

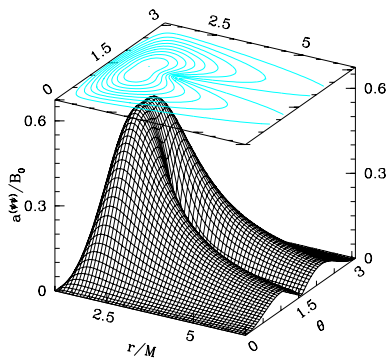

What is a Killing Vector?

A Killing vector points in a direction in spacetime that you can move without the spacetime changing locally. A Killing vector encodes a symmetry. 


\section{Field and Photons}

To understand the interaction of light with the magnetized vacuum, we imagined expanding the action for a uniform field plus a small photon field,

$$
\mathbf{E}=\mathbf{E}_{0}+\delta \mathbf{E}, \mathbf{B}=\mathbf{B}_{0}+\delta \mathbf{B}, F^{\mu \nu}=\left(F_{0}\right)^{\mu \nu}+f^{\mu \nu} .
$$

We have two possibilities.

1. $k \lambda_{e} \ll 1$ : we pretend that the photon field is also uniform and expand the effective Lagrangian density.

2. $k \lambda_{e} \gtrsim 1$ : we have to expand the action itself. 


\section{How It Works}

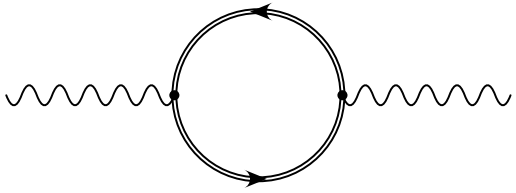

$S=S_{0}+\frac{1}{2} f^{\mu \nu} f^{\alpha \beta} \frac{\delta^{2} S}{\delta f^{\mu \nu} \delta f^{\alpha \beta}}$

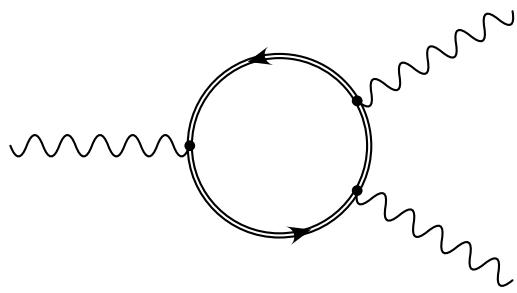

$S=S_{0}+\frac{1}{2} f^{\mu \nu} f^{\alpha \beta} \frac{\delta^{2} S}{\delta f^{\mu \nu} \delta f^{\alpha \beta}}$ 


\section{Indices and Indices, Oh My!}

It would appear that we have an explosion of terms. However, we can make several important simplifications.

- The Lagrangian density and the action are gauge invariant, so they can only depend on gauge invariant quantities, i.e. $F^{\mu \nu}$.

- The Lagrangian density and the action are Lorentz invariant, so they can only depend on Lorentz scalars.

- If the field and the perturbation can be taken to be uniform $\left(k^{\mu} \rightarrow 0\right)$, then we can use the Lagrangian density which can only depend on $I=F^{\mu \nu} F_{\mu \nu}=2\left(b^{2}-a^{2}\right)$ and $K=J^{2}=\left(\epsilon_{\mu \nu \alpha \beta} F^{\mu \nu} F^{\alpha \beta}\right)^{2}=(-4 a b)^{2}$ 


\section{The Lagrangian Density}

We already saw an expression for the Lagrangian density for arbitrary uniform fields,

$$
\mathcal{L}_{\text {eff }}=\frac{\hbar}{8 \pi^{2}} B_{k}^{2} \int_{0}^{\infty} \frac{d \zeta}{\zeta} e^{-i \zeta}\left[\frac{a b}{B_{k}^{2}} \operatorname{coth}\left(\zeta \frac{a}{B_{k}}\right) \cot \left(\zeta \frac{b}{B_{k}}\right)-\mathrm{CT}\right] .
$$

As is this is a complex integral and the integrand is a bit poorly behaved. The integral converges through a cancellation with the counterterms. Because we are interested in uniform magnetic fields with small perturbations we can perform a Wick rotation, yielding

$$
\mathcal{L}_{\text {eff }}=\frac{\alpha}{8 \pi^{2}} B_{k}^{2} \int_{0}^{\infty} \frac{d \zeta}{\zeta} e^{-\zeta}\left[-\frac{a b}{B_{k}^{2}} \cot \left(\zeta \frac{a}{B_{k}}\right) \operatorname{coth}\left(\zeta \frac{b}{B_{k}}\right)+\mathrm{CT}\right]
$$




\section{The Lagrangian Density}

$$
\mathcal{L}_{\text {eff }}=\frac{\alpha}{8 \pi^{2}} B_{k}^{2} \int_{0}^{\infty} \frac{d \zeta}{\zeta} e^{-\zeta}\left[-\frac{a b}{B_{k}^{2}} \cot \left(\zeta \frac{a}{B_{k}}\right) \operatorname{coth}\left(\zeta \frac{b}{B_{k}}\right)+\mathrm{CT}\right]
$$

This expression is manifestly real, so if $a \neq 0$, this does not give a valid expression for the imaginary part of the action. However, this expression yields some useful simplifications.

Let us write an expansion for small values of $K$ Heyl, Hernquist 97a

$$
\mathcal{L}_{\text {eff }}=\frac{e^{2}}{h c} \xi^{2} B_{k}^{2} X_{0}\left(\frac{1}{\xi}\right)+\frac{e^{2}}{h c} \frac{K}{B_{k}^{2}} X_{1}\left(\frac{1}{\xi}\right)+\frac{e^{2}}{h c} \frac{K^{2}}{B_{k}^{2}} X_{2}\left(\frac{1}{\xi}\right)+\cdots
$$

for nearly wrenchless fields where $\xi^{2}=\left(b^{2}-a^{2}\right) / B_{k}^{2}$. 


\section{The Lagrangian Density}

$$
\mathcal{L}_{\text {eff }}=\frac{e^{2}}{h c} \frac{I}{2}\left[X_{0}\left(\frac{1}{\xi}\right)+\frac{K}{8 I^{2}} X_{1}\left(\frac{1}{\xi}\right)+\frac{K^{2}}{384 I^{4}} X_{2}\left(\frac{1}{\xi}\right)+\cdots\right]
$$

where

$$
\begin{gathered}
X_{0}(x)=4\left[\int_{1}^{x / 2+1} \ln \Gamma(v) d v-\ln A\right]-\frac{1}{3} \ln \left(\frac{x}{2}\right)+x[1-\ln (\pi x)] \\
+x^{2}\left[\frac{3}{4}-\frac{1}{2} \ln \left(\frac{x}{2}\right)\right], \ln A=\frac{1}{12}-\zeta^{(1)}(-1)=0.248754477
\end{gathered}
$$




\section{The Mother Function, $X_{0}(x)$}

Some neat facts about $X_{0}(x)$,

- For pure electric fields, the value of $\xi$ is imaginary and $X_{0}(x)$ is complex and gives the value of the Lagrangian density before the Wick rotation, even the imaginary part.

- The derivatives of the Lagrangian density with respect to $K$, $X_{1}(x)$ and $X_{2}(x)$, can be expressed as derivatives of $X_{0}(x)$.

- These functions are all that you need to understand photons of long wavelength travelling through a wrenchless field.

- It is actually easier to calculate for strong fields than for weak ones and is related to zeta-function regularization. 


\section{Functions}

$$
\begin{aligned}
X_{0}(x)= & 4\left[\int_{1}^{x / 2+1} \ln \Gamma(v) d v-\ln A\right]-\frac{1}{3} \ln \left(\frac{x}{2}\right)+x[1-\ln (\pi x)] \\
+ & x^{2}\left[\frac{3}{4}-\frac{1}{2} \ln \left(\frac{x}{2}\right)\right], \ln A=\frac{1}{12}-\zeta^{(1)}(-1)=0.248754477 . \\
X_{1}(x)= & -2 X_{0}(x)+x X_{0}^{(1)}(x)+\frac{2}{3} X_{0}^{(2)}(x)-\frac{2}{9} \frac{1}{x^{2}} \\
X_{2}(x)= & -24 X_{0}(x)+9 x X_{0}^{(1)}(x)+\left(8+3 x^{2}\right) X_{0}^{(2)}(x)+4 x X_{0}^{(3)}(x) \\
& -\frac{8}{15} X_{0}^{(4)}(x)+\frac{8}{15} \frac{1}{x^{2}}+\frac{16}{15} \frac{1}{x^{4}}
\end{aligned}
$$




\section{Index of Refraction}

For light travelling through a strong magnetic field there are two possibilities

1. The parallel mode (following the convention of Adler) where $\delta \mathbf{B} \| \mathbf{B}$, so $\delta I=8 B \delta B$ and $\delta J=0$, and

2. The perpendicular mode where $\delta \mathbf{B} \perp \mathbf{B}$, so $\delta l=0$ and $\delta J=-4 B \delta E$.

3. Because $\partial^{2} \mathcal{L}_{\mathrm{Eff}} / \partial I \partial J=0$ for wrenchless fields, these are the modes of propagation. 


\section{Index of Refraction}

Looking at these two modes for light travelling through a strong magnetic field yields the following expressions for the index of refraction,

$$
\begin{gathered}
n_{\perp}=1-\frac{\alpha}{4 \pi} X_{1}\left(\frac{1}{\xi}\right) \sin ^{2} \theta \\
n_{\|}=1+\frac{\alpha}{4 \pi}\left[X_{0}^{(2)}\left(\frac{1}{\xi}\right) \xi^{-2}-X_{0}^{(1)}\left(\frac{1}{\xi}\right) \xi^{-1}\right] \sin ^{2} \theta
\end{gathered}
$$

Potehkin et al. have derived convenient fitting formulae for these values that are valid to within a $3 \%$ for all field strengths

$$
n_{\perp}=1+\frac{7 \alpha}{90 \pi} \xi^{2} \frac{1+1.2 \xi}{1+1.35 \xi+0.56 \xi^{2}}, n_{\|}=1+\frac{\alpha}{6 \pi} \frac{\xi^{2}}{3.75+2.7 \xi^{5 / 4}+\xi^{2}}
$$

Heyl, Hernquist 97b 


\section{Index of Refraction}

What could be a signature of this birefringence?

- A time delay: $\Delta t \sim$ $10^{-3} \mathrm{R} / \mathrm{c} \sim 10 \mathrm{~ns}$ ?

- Magnetic lensing? Shaviv et al. 99

- These all seemed a bit too subtle.

- We were literally staring at the answer.

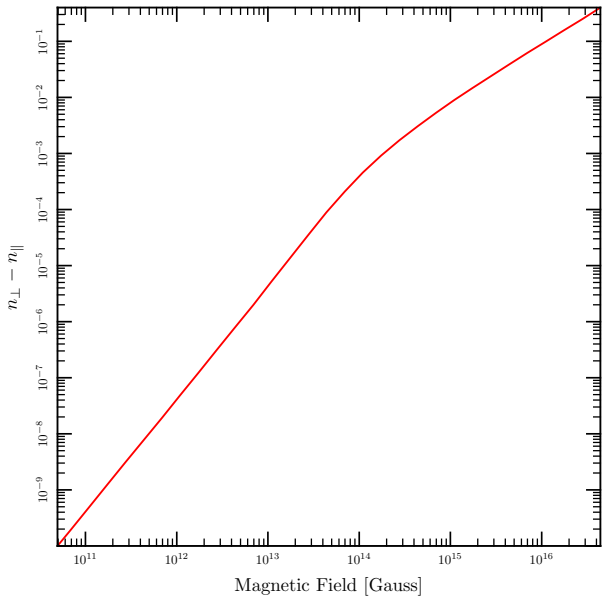




\section{Propagation through a twisting magnetic field}

Kubo and Nagata (1983) present a concise way to characterize the evolution of the polarization of light through a medium. Normally one represents this by complex amplitudes; however, one can exploit the homeomorphism between $\mathrm{SU}(2)$ and $\mathrm{SO}(3)$ to represent light using the four Stokes parameters.

$$
\frac{\partial \mathbf{s}}{\partial l}=\hat{\mathbf{\Omega}} \times \mathbf{s}
$$

where $|\hat{\boldsymbol{\Omega}}|=\Delta k$. The vector $\mathbf{s}=\left(S_{1}, S_{2}, S_{3}\right) / S_{0}$ or $(Q, U, V) / I$. 


\section{Stokes Parameters and the Poincaré Sphere}

An important analytic solution.

What if $\frac{\partial \hat{\boldsymbol{\Omega}}}{\partial l}=\hat{\boldsymbol{\Upsilon}} \times \hat{\boldsymbol{\Omega}}$ ?

1. Move into frame that corotates with $\hat{\Omega}$.

2. In this frame we have

$$
\frac{\partial \mathbf{s}}{\partial l}=(\hat{\boldsymbol{\Omega}}-\hat{\boldsymbol{\gamma}}) \times \mathbf{s}
$$

3. s orbits $\hat{\Omega}_{\mathrm{Eff}}$ if

$$
\left|\hat{\mathbf{\Omega}}\left(\frac{1}{|\hat{\mathbf{\Omega}}|} \frac{\partial|\hat{\mathbf{\Omega}}|}{\partial /}\right)^{-1}\right| \gtrsim 0.5
$$




\section{Polarization-Limiting Radius}

The radius at which the polarization stops following $\hat{\Omega}$ is called the polarization-limiting radius. Beyond here the modes are coupled. It is safe to assume that this occurs in the weak-field limit where

$$
|\hat{\mathbf{\Omega}}|=\frac{\alpha}{4 \pi} \frac{2}{15}\left(\frac{B}{B_{\mathrm{QED}}}\right)^{2} \frac{c}{\omega} \sin ^{2} \theta,\left.|\hat{\mathbf{\Omega}}| \frac{1}{|\hat{\mathbf{\Omega}}|} \frac{\partial|\hat{\mathbf{\Omega}}|}{\partial I}\right|^{-1} \mid=\Delta n \frac{c}{\omega} \frac{r}{6}
$$

Therefore, the polarization-limiting radius is

$$
\begin{aligned}
r \lesssim r_{\mathrm{pl}} & \equiv\left(\frac{\alpha}{45} \frac{\nu}{c}\right)^{1 / 5}\left(\frac{\mu}{B_{\mathrm{QED}}} \sin \beta\right)^{2 / 5} \\
& \approx 1.2 \times 10^{7}\left(\frac{\mu}{10^{30} \mathrm{Gcm}^{3}}\right)^{2 / 5}\left(\frac{\nu}{10^{17} \mathrm{~Hz}}\right)^{1 / 5}(\sin \beta)^{2 / 5} \mathrm{~cm}
\end{aligned}
$$


Outline

Objects

Processes

Magnetars

Observations
Vacuum Breakdown

Vacuum Polarization

Axions and Plasmas

Photon Splitting

\section{Why does this matter?}

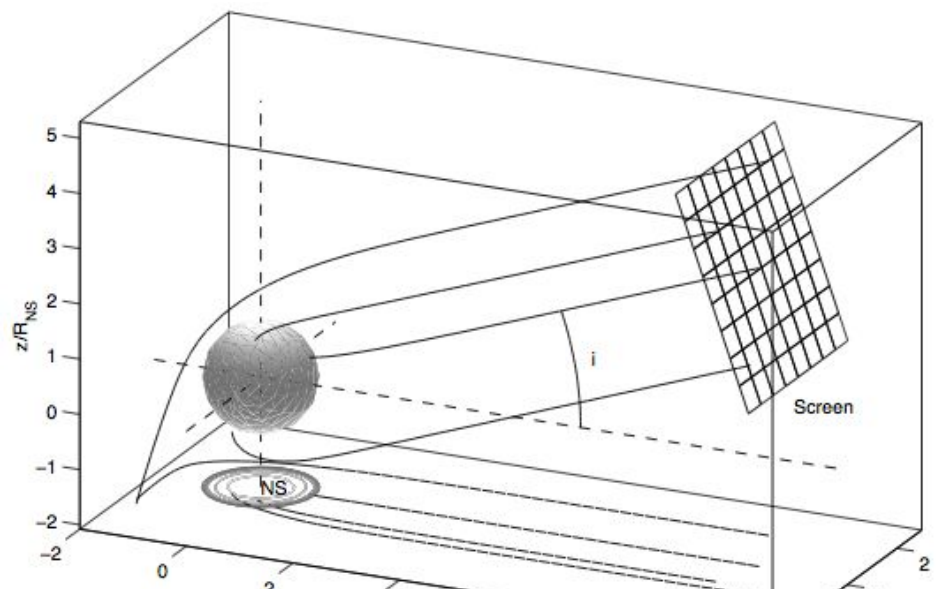




\section{Why does this matter?}
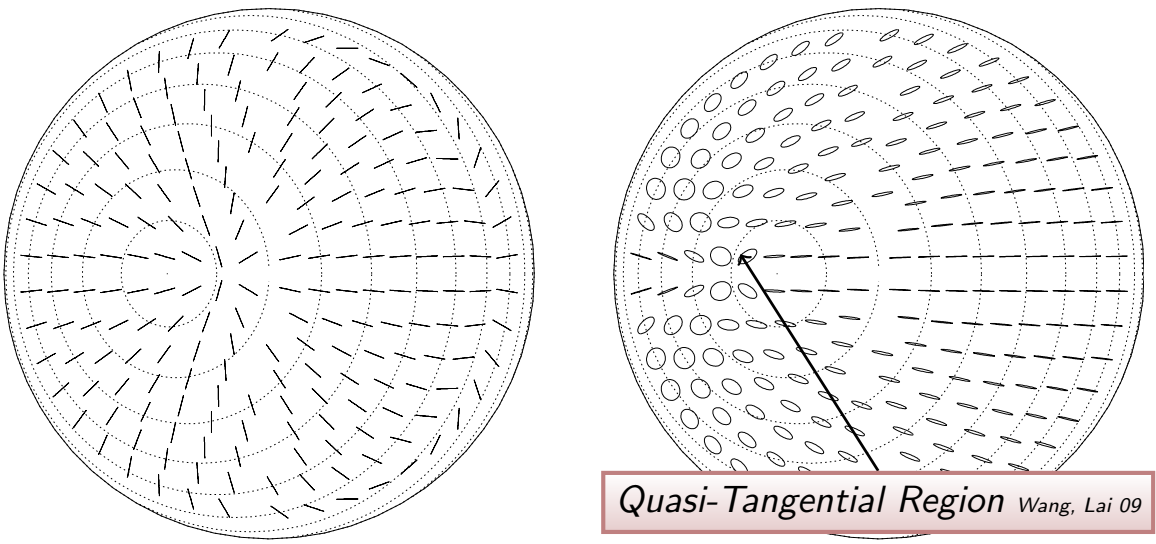

Heyl, Shaviv 02 


\section{This is not subtle.}

Let's recap.

- Neutron star atmospheres emit polarized light.

- The emission varies across the surface.

- The rotating magnetic field twists the polarization.

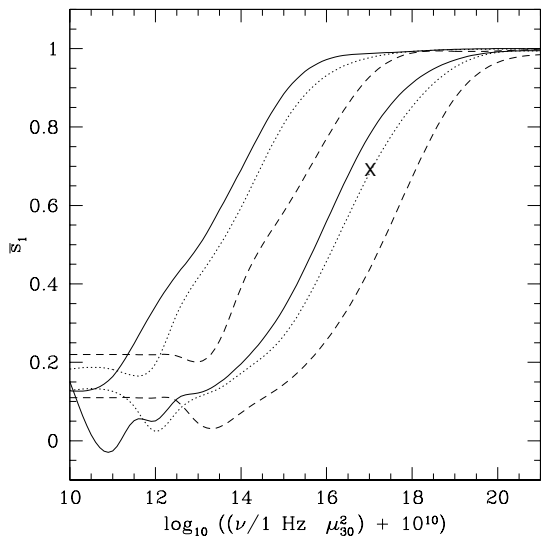




\section{Realistic Hydrogen Atmosphere}

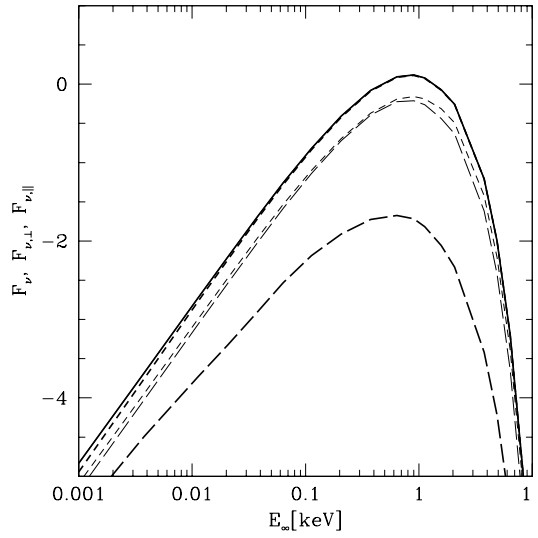

$10^{12} \mathrm{G}$

Heyl, Shaviv, Lloyd 05

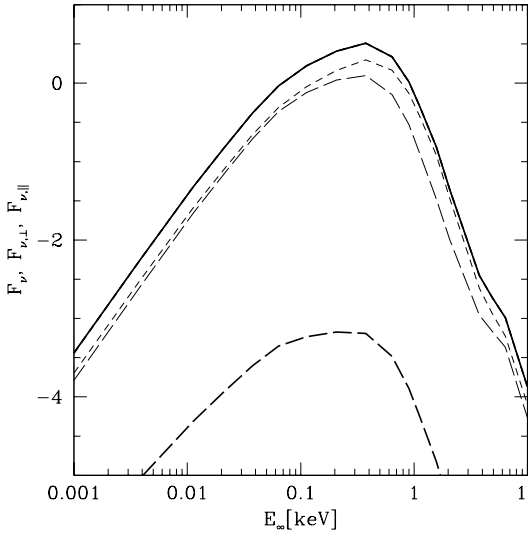

$10^{14} \mathrm{G}$ 


\section{Axions and Plasmas}

Let's add an axion which is a pseudoscalar particle that can couple to a photon travelling through a magnetic field. $\mathcal{L}_{a \gamma \gamma}=-\frac{1}{4} g$ a $F_{\mu \nu} \tilde{F}^{\mu \nu}=g$ a $\mathbf{E} \cdot \mathbf{B}$

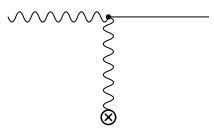

$$
i c \frac{d}{d z}\left[\begin{array}{c}
a \\
E_{\|} \\
E_{\perp}
\end{array}\right]=\left[\begin{array}{ccc}
\omega+\Delta_{a} & \Delta_{M} & 0 \\
\Delta_{M} & \omega+\Delta_{\|}+\Delta_{p} & \sigma_{12} \omega / 2 \\
0 & \sigma_{21} \omega / 2 & \omega+\sigma_{22} \omega / 2
\end{array}\right]\left[\begin{array}{c}
a \\
E_{\|} \\
E_{\perp}
\end{array}\right] \text {, }
$$

where using $\beta=B \sin \theta / B_{\mathrm{QED}}$ we have the $\mathrm{QED}$ and axion contributions

$\Delta_{\|}=\frac{7 \alpha}{90 \pi} \hat{q} \beta^{2} \omega, \Delta_{a}=-\frac{m_{a}^{2}}{2 \omega}, \Delta_{M}=\frac{g m_{e}^{2}}{2} \beta, \sigma_{22}=\frac{4 \alpha}{45 \pi} \beta^{2} \hat{m}+\sigma_{22, p}$ 


\section{Three-State Equation}

$$
i c \frac{d}{d z}\left[\begin{array}{c}
a \\
E_{\|} \\
E_{\perp}
\end{array}\right]=\left[\begin{array}{ccc}
\omega+\Delta_{a} & \Delta_{M} & 0 \\
\Delta_{M} & \omega+\Delta_{\|}+\Delta_{p} & \sigma_{12} \omega / 2 \\
0 & \sigma_{21} \omega / 2 & \omega+\sigma_{22} \omega / 2
\end{array}\right]\left[\begin{array}{c}
a \\
E_{\|} \\
E_{\perp}
\end{array}\right] \text {, }
$$

where using $\beta=B \sin \theta / B_{\mathrm{QED}}$ we have $\mathrm{QED}$, axion and plasma terms

$$
\begin{gathered}
\Delta_{\|}=\frac{7 \alpha}{90 \pi} \hat{q} \beta^{2} \omega, \Delta_{a}=-\frac{m_{a}^{2}}{2 \omega}, \Delta_{M}=\frac{g m_{e}^{2}}{2} \beta, \sigma_{22}=\frac{4 \alpha}{45 \pi} \beta^{2} \hat{m}+\sigma_{22, p} \\
\Delta_{p}=-\frac{\omega v_{e}}{2}\left[\frac{\cos ^{2} \theta}{1-u_{e}}+\sin ^{2} \theta\right], \sigma_{12}=-\sigma_{21}=i \frac{v_{e} u_{e}^{1 / 2}}{1-u_{e}} \cos \theta, \sigma_{22, p}=-\frac{v_{e}}{1-u_{e}}
\end{gathered}
$$

where $u_{e}=\omega_{c e}^{2} / \omega^{2}, v_{e}=\omega_{p e}^{2} / \omega^{2}, \omega_{c e}=m_{e} c^{2} \xi / \hbar$ and $\omega_{p e}=\left(4 \pi n_{e} e^{2} / m_{e}\right)^{1 / 2}$. The values of $\hat{m}$ and $\hat{q}$ are of order unity for small fields and

$$
\hat{q}=\frac{1+1.2 \xi}{1+1.33 \xi+0.56 \xi^{2}}, \hat{m}=\frac{1}{1+0.72 \xi^{5 / 4}+(4 / 15) \xi^{2}} .
$$




\section{Resonances}

If the second and third diagonal elements are equal, we have a resonance between the two photon modes.

If the first and second diagonal elements are equal, we have a resonance between the photon and the axion.

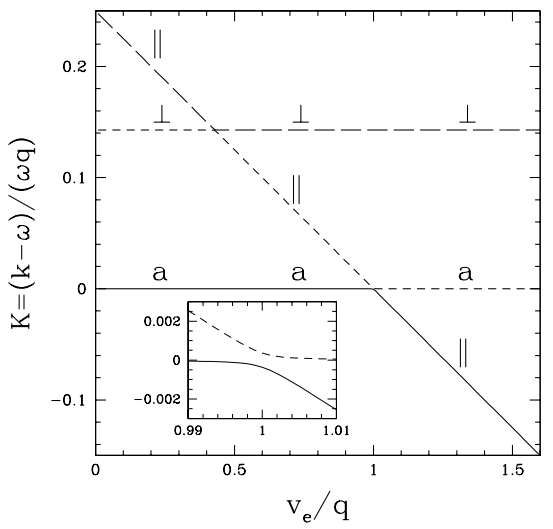




\section{Vacuum-Plasma Resonance}

Deep in the atmosphere of the neutron star the plasma dominates, while outside the vacuum dominates.

The transition is adiabatic if

$$
\begin{aligned}
E \gtrsim E_{\mathrm{ad}}= & 2.52\left(f \tan \theta\left|1-u_{i}\right|\right)^{2 / 3} \times \\
& \left(\frac{1 \mathrm{~cm}}{H_{\rho}}\right)^{1 / 3} \mathrm{keV}
\end{aligned}
$$

and the conversion probability is

$$
P_{\text {con }}=1-\exp \left[-(\pi / 2)\left(E / E_{\text {ad }}\right)^{3}\right] \text {. }
$$

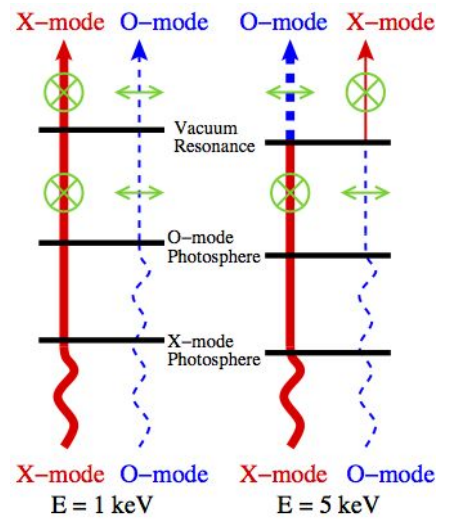

Lai, Ho 03 


\section{Vacuum-Plasma Resonance}

For large strengths of the magnetic field, the vacuum resonance may lie between the photospheres

$$
B \gtrsim B_{I} \approx 6.6 \times 10^{13} T_{6}^{-1 / 8} E_{1}^{-1 / 4} S^{-1 / 4} \mathrm{G}
$$

where $S=1-e^{-E / k T}$.

This can have a strong effect on the appearance of spectral features and the high-energy slope. Ho, Lai 04

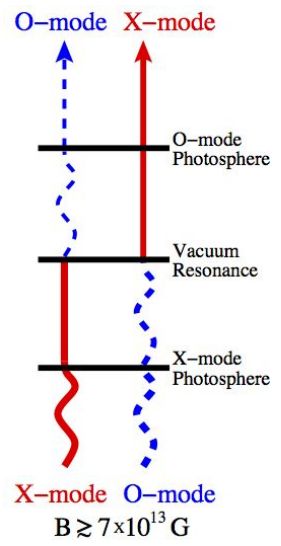




\section{Vacuum-Plasma Resonance}
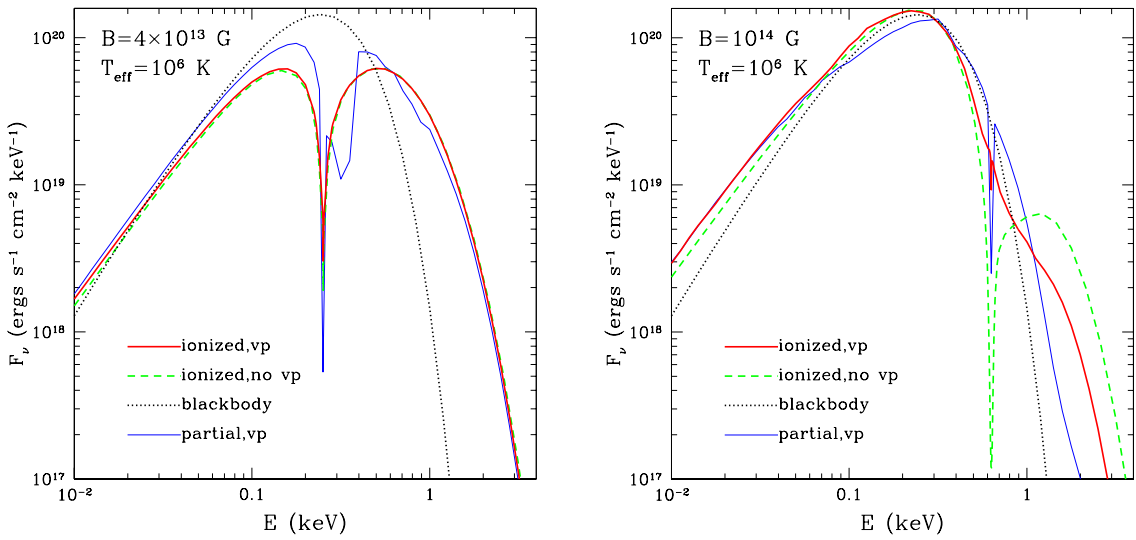

$B=4 \times 10^{13} \mathrm{G}$

$B=10^{14} \mathrm{G}$

Ho, Lai 04 


\section{Axion-Photon Resonance}

With generous parameters for the axion $\left(g=10^{-6} \mathrm{GeV}^{-1}\right)$, $\left.m_{a}=10^{-3} \mathrm{eV}\right)$, we can get substantial conversion to the axion, resulting in strongly polarized light.

However, we already expect the neutron atmospheres to be strongly polarized, so it wouldn't be a clear signature.

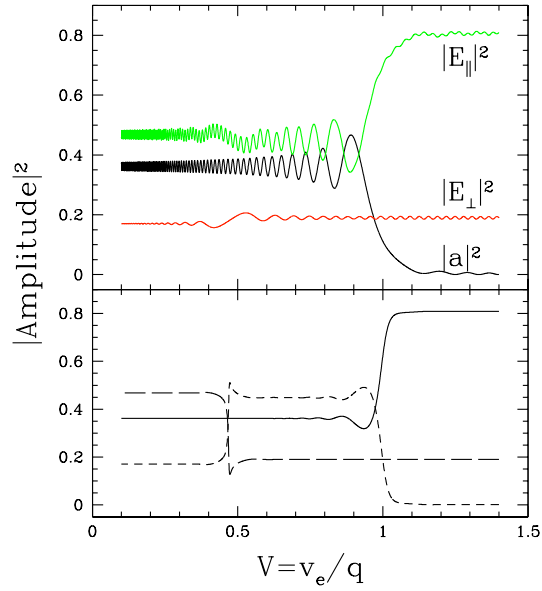

Lai, Heyl 06; Perna et al 12 


\section{Axions and White Dwarfs}

White dwarfs have exquisite polarimetric observations, finding no linear polarization to the few percent level.

Their fields are weaker $10^{8-9} \mathrm{G}$, but the stars are bigger, and we know the field geometry.

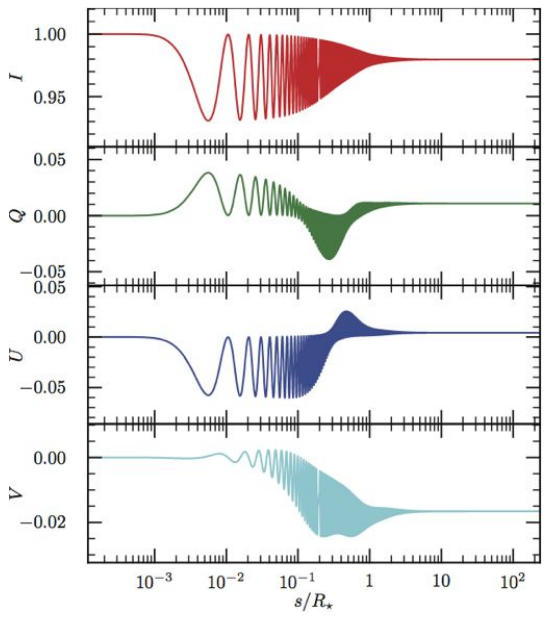

Gill, Heyl 11 


\section{Axions and White Dwarfs}

Assuming that we model the field in the gigagauss white dwarfs,

PG $1031+234$ and SDSS J234605+385337 as well as Euchner et al 2006 did for PG 1015+015, the observed minimum polarization of a few percent excludes some of the currently allowed region for axion-like particles.

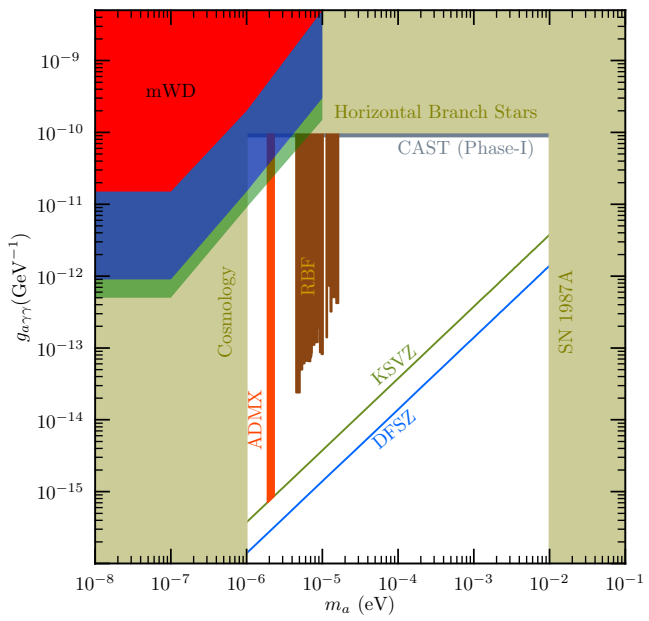

Gill, Heyl 11 


\section{Photon Splitting}

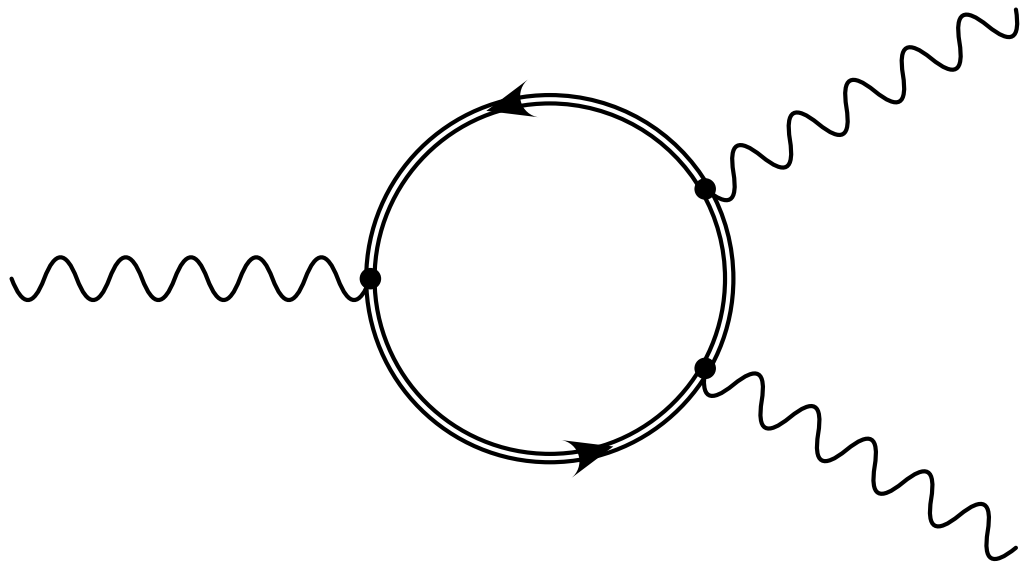




\section{Photon Splitting Cascades}

In a neutron star magnetosphere, only one splitting process is permitted

$$
\| \rightarrow \perp+\perp
$$

because the index of refraction of the parallel mode is greater than the perpendicular mode, so this is the only process that conserves momentum and $C P$.

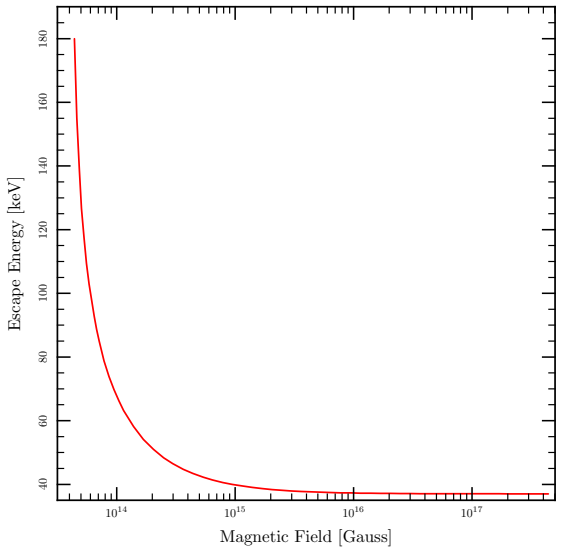

Heyl, Hernquist 97b 


\section{Photon Splitting Cascades}

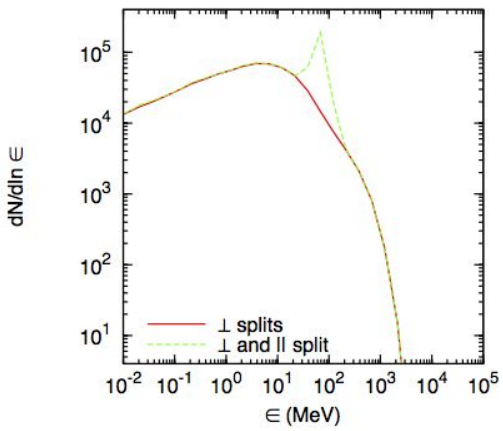

Photon Spectra

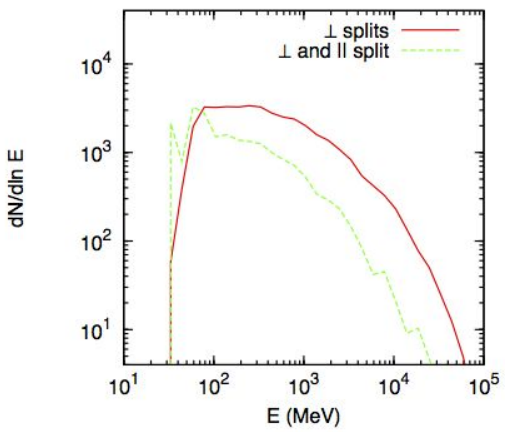

Pair Spectra

Medin, Lai 2010 


\section{Photon Splitting - Gamma Rays}

Photon splitting has often been invoked to explain hard cutoffs in gamma-ray spectra from pulsars and GRBs. However, these models are difficult to interpret because we don't know where the photons are actually produced.

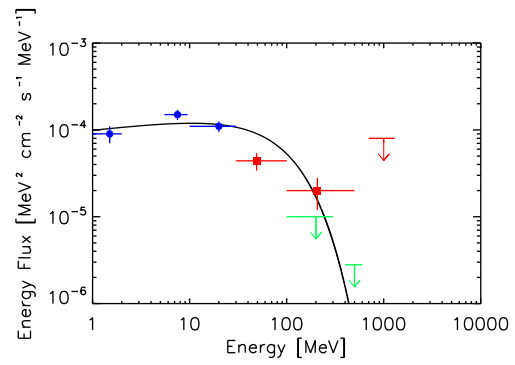




\section{The Magnetars - Why?}

There are several lines of evidence for the strong fields of magnetars in addtion to the spin down.

- Energetics $\left(10^{40} \mathrm{~W}\right.$ over $0.1 \mathrm{~s})$,

- Timescales (bursts),

- Cooling (field decay).

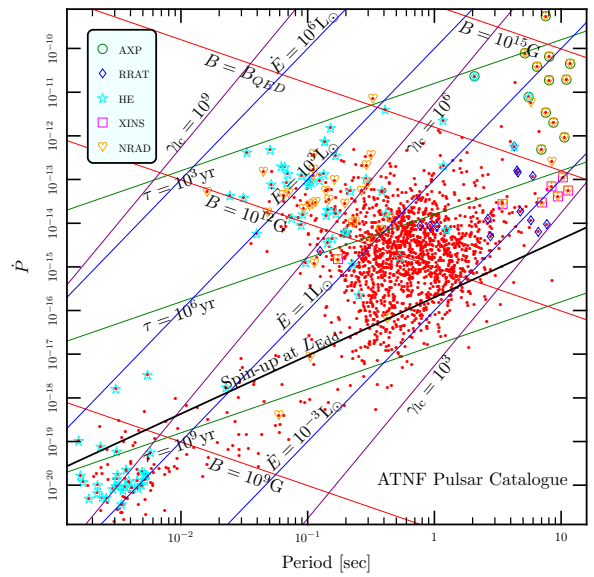




\section{What are magnetars?}

- Neutron stars with magnetic fields larger, than $B_{\mathrm{QED}}=m^{2} c^{3} /(e \hbar) \approx 4.4 \times 10^{13} \mathrm{G}$,

- Magnetically powered neutron stars,

- Isolated neutron stars that exhibit x-ray flares (SGRs)

- Isolated neutron stars that are too hot for their age,

- All of these criteria play a role. 


\section{Extreme Physics}

- Magnetic stresses exceed yield stress of the crust

(Thompson \& Duncan '95)

- Strongly distorted atoms

(Thirumalai \& Heyl '09)

- Radiative corrections of QED (Thompson, Heyl, Lai, etc.)

- Magnetar quakes (Strohmayer, Watts, etc.)
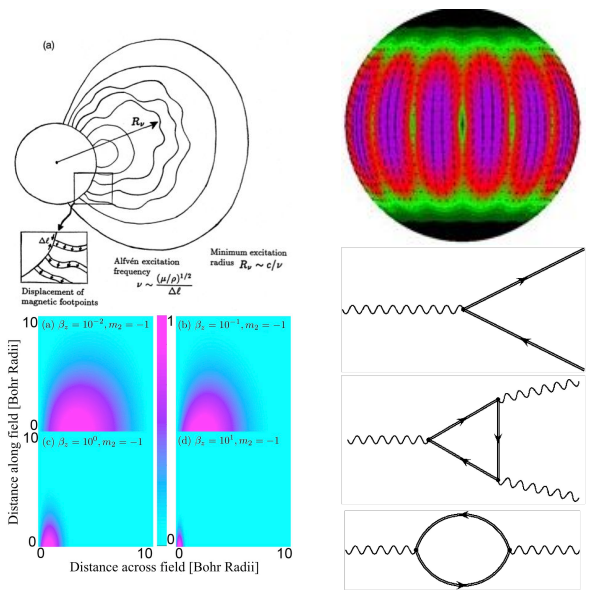


\section{How does the field evolve?}

The strong magnetic field is implicated in "magnetar" phenomena.

- Gradual decay of the magnetic field powers the quiescent emission, (Heyl \& Kulkarni '98; Thompson \& Duncan '96)

- As the crust cracks the magnetic field suddenly shifts, powering the flares.

(Thompson \& Duncan '95)
Modes of magnetic field decay

Goldreich \& Reisenegger '92

- Ohmic decay $t_{\mathrm{Ohm}} \approx 2 \times 10^{11} \frac{L_{5}^{2}}{T_{8}^{2}} \mathrm{yr}$

- Hall drift $t_{\mathrm{Hall}} \approx 5 \times 10^{8} \frac{L_{5}^{2} T_{8}^{2}}{B_{12}} \mathrm{yr}$

- Ambipolar diffusion $t_{\mathrm{irr}, \mathrm{amb}} \approx 5 \times 10^{15} \frac{1}{T_{8}^{6} B_{12}^{2}} \mathrm{yr}$ $t_{\mathrm{sol}, \mathrm{amb}} \approx 3 \times 10^{9} \frac{L_{5}^{2} T_{8}^{2}}{B_{12}^{2}} \mathrm{yr}$ 


\section{Evidence of magnetic field decay}

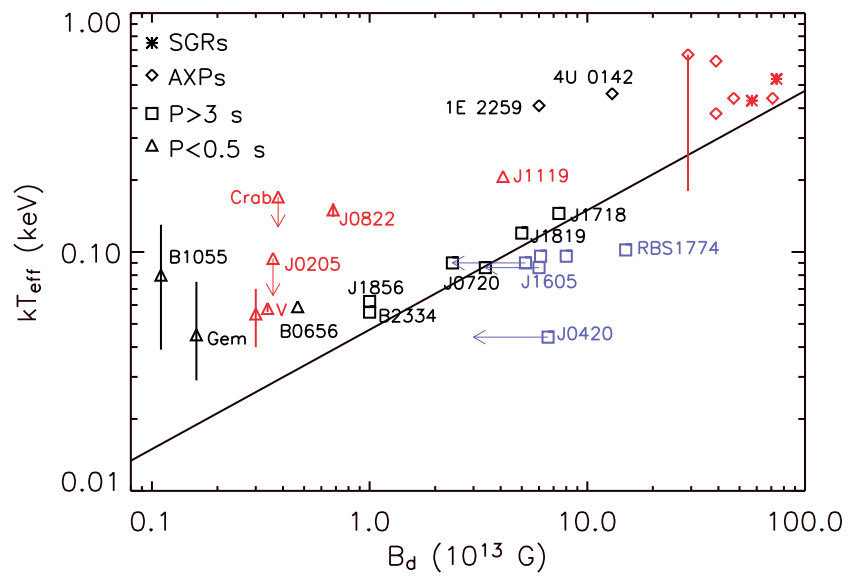




\section{Flares}

- And they have the biggest repeating explosions in the universe!

- The explosions are so big that they reionize the Earth's atmosphere.

They have a huge spike of radiation plus a periodic signal of a few seconds as the star cools.

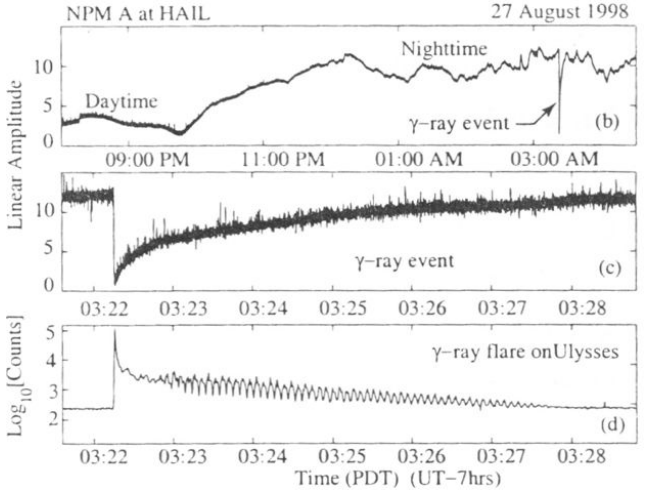

Inan et al. '99 


\section{Flares}

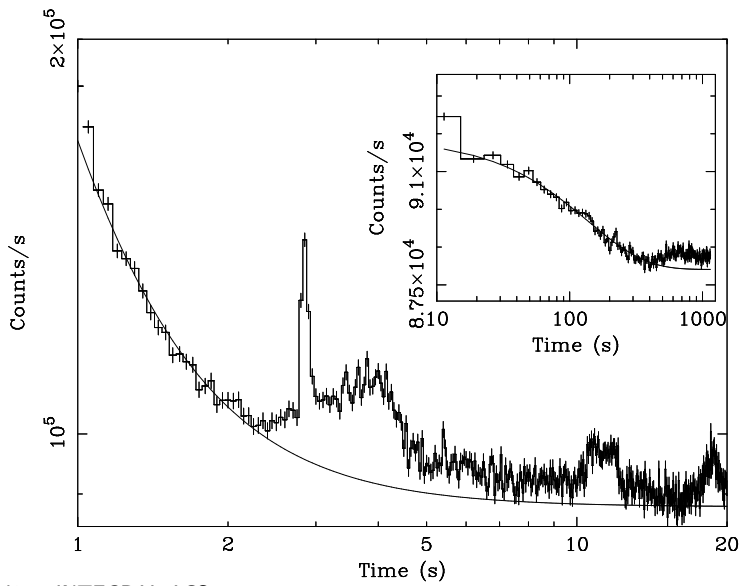

Mereghetti et al. '05 - INTEGRAL ACS 


\section{Flares}

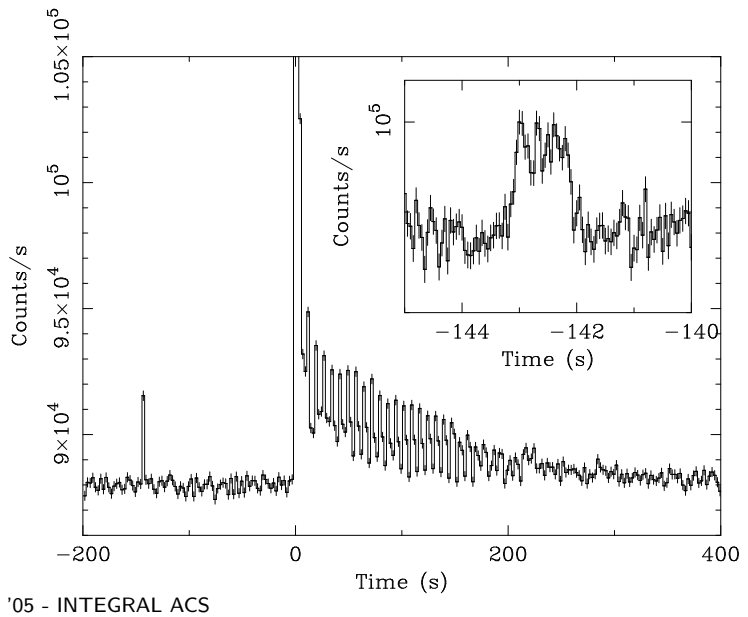

Mereghetti et al. '05 - INTEGRAL ACS 


\section{That was the bake. Now comes the shake.}

As the star cools, the emission oscillates at many frequencies.

- $92 \mathrm{~Hz}$

- $625 \mathrm{~Hz}$

- $1840 \mathrm{~Hz}$

- Probes the properties of the crust.

- Higher pressure, higher frequency

- Higher density, lower frequency

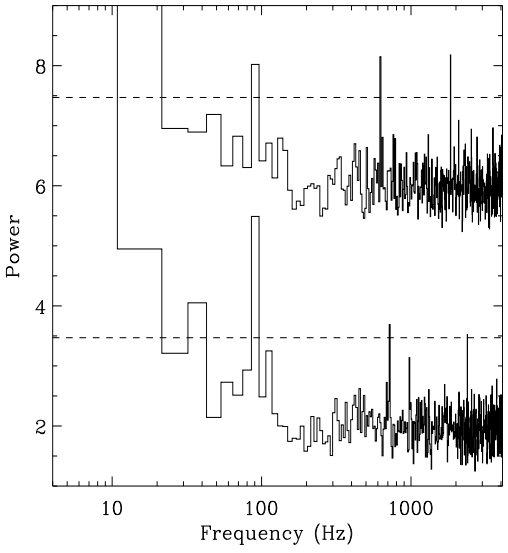

Watts, Reddy '07 


\section{That was the bake. Now comes the shake.}

- Watts and Reddy '07 concluded that a thin, strange star crust is probably excluded.

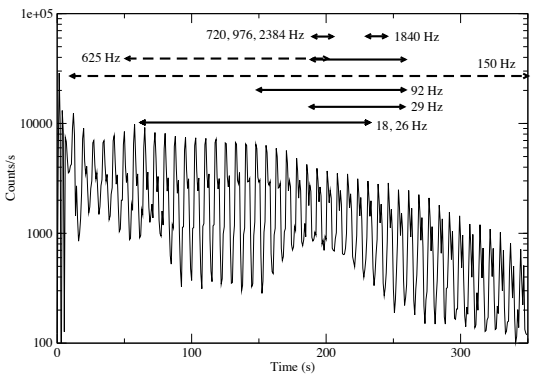

Watts, Reddy '07 


\section{What if it doesn't crack?}

What made us think that the crust was cracking in the first place?

Statistics of SGR flares are similar to earthquakes.

Statistics of solar flares are similar to earthquakes. 


\section{Plasma Physics}

(a). Precursor Phase

Reconnection Region

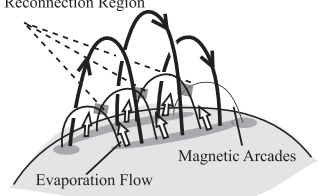

(b). Quiescent Phase

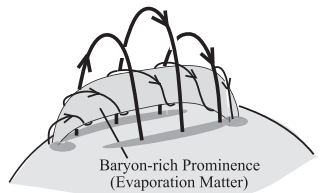

(c). Main Burst Phase

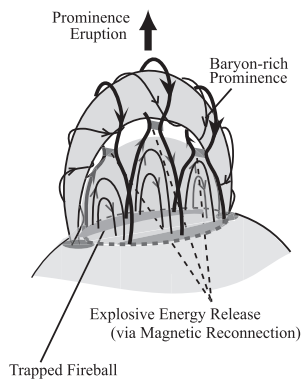

(d). Mass Ejection Phase

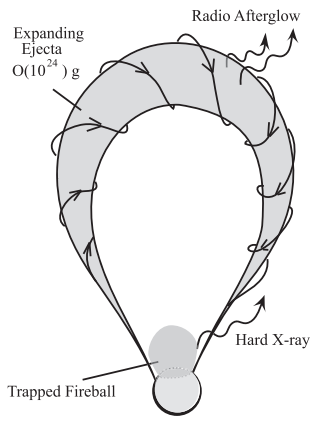

Masada et al. '08, Lyutikov '06 


\section{SGR Precursors}

The SGR hyperflares each have been preceded by a small flare. Can the time between the two flares tell us anything? Gill, Heyl 10

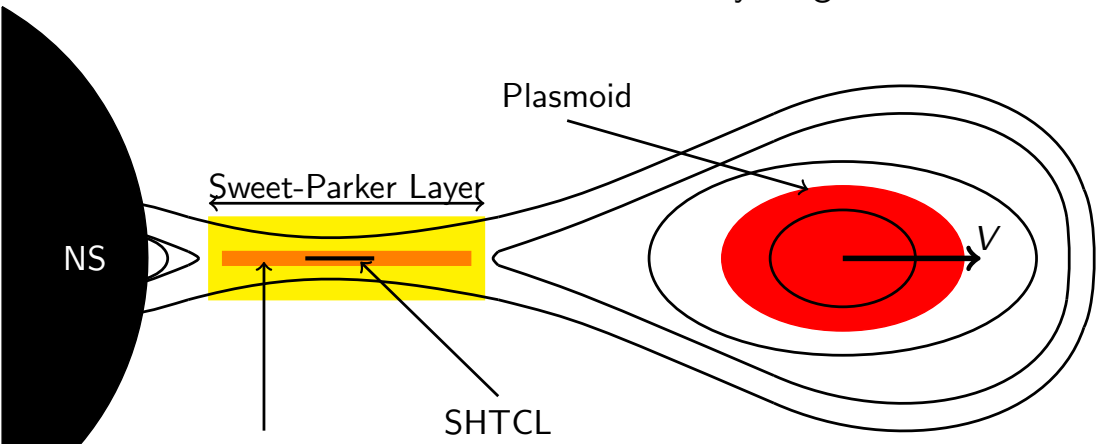
Hall Reconnection Layer 


\section{What comprises the surface layers? (Atoms/Molecules)}

The atomic physics complicates the interpretation of the spectra from these neutron stars. At $3 \times 10^{13} \mathrm{G}$, helium molecules (and chains) could be the dominant species in the cool atmospheres of high-B pulsars. At $2 \times 10^{14} \mathrm{G}$, atomic helium dominates for both magnetars and high-B pulsars. Mori \& Heyl '07
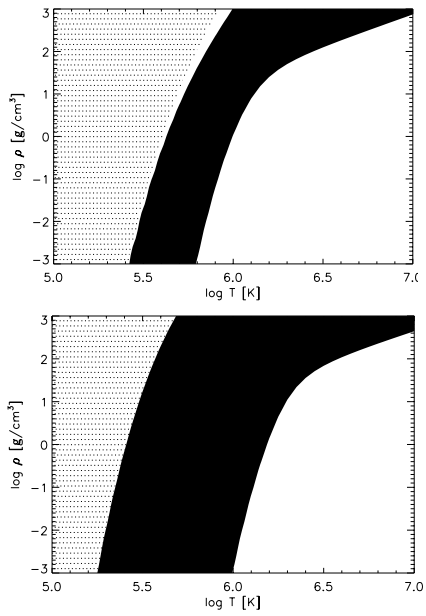


\section{What comprises the surface layers? $(\mathrm{H}, \mathrm{He}, \mathrm{Fe}$,other $)$}

At the crust of the neutron star cools over the first year, nuclear reactions freeze out before nuclear statistical equilibrium is reach. The crust is not "cold catalyzed nuclear matter" but contains significant impurities.

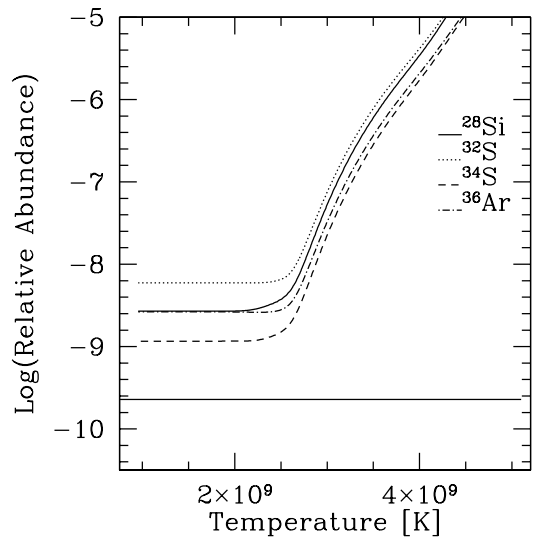

Hoffman \& Heyl '09 


\section{What produces the radiation?}

- Thermal radiation from the surface is supplied by initial heat and field decay $\left(U \approx \mathcal{M}\right.$ at $10^{3}$ years and $\left.10^{15} \mathrm{G}\right)$. JH et al. '97b, '98, '05

- Currents in the twisted magnetosphere generate hard $\mathrm{x}$-ray emission through resonant Compton scattering. Thompson '08; Rea et al. '08; Nobili et al. '08

- MHD fast modes can produce non-thermal emission through the formation of QED-catalyzed shocks. JH et al. '05b, '07; Brodin et al. '07
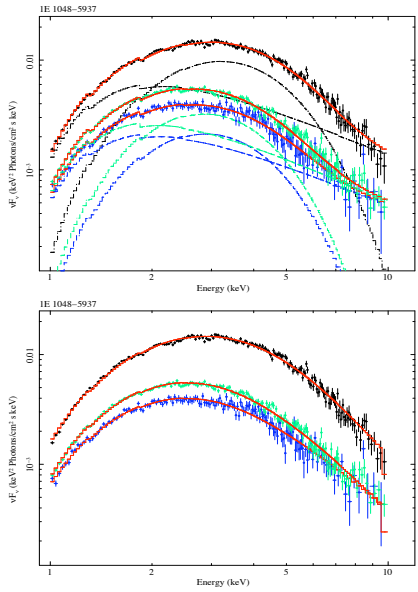


\section{To be II or not to be II? (I)}

- Neutron superfluid and proton Type-II superconductor cannot coexist in a star with long-period precession.

What makes a material Type I or Type II? Link '03

- If the flux tubes carry a Do the flux tubes repel or attract each other? persistent current, you get Type-I Charbonneau \& Zhitnitsky ' 07.

- What does QED say about this? Mazur, Heyl 


\section{To be II or not to be II? (II)}

Type I

Meet the intermediate state

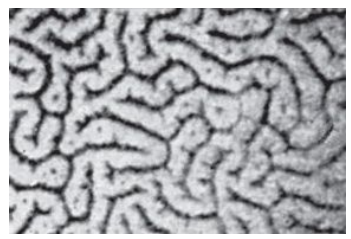

The Open University

If the domains are small they will move with the vortex lines; otherwise, they will move as a normal charged Fermi system.

\section{Type II}

Can the flux tubes move relative to the fluid?

- Yes Jones '06a

- Not if hot Jones '09

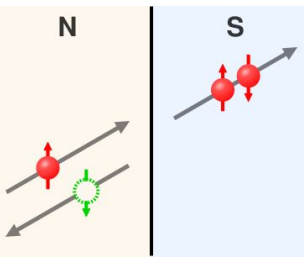

Andreev Reflection (Wikipedia) 


\section{What don't we know?}

- Why are only some high-field neutron stars "magnetars"?

- Why are "magnetars" hot?

- How does the crust break? Does it break?

- How does the field decay? Or grow?

This list is not exhaustive. 


\section{GEMS}

- Gravity and Extreme Magnetism SMEX

- To be launched after November 2014

- 35 prime targets, including stellar black holes, seyfert galaxies and quasars, blazars, a variety of neutron stars including pulsars and magnetars, shell supernova remnants and pulsar wind nebulae

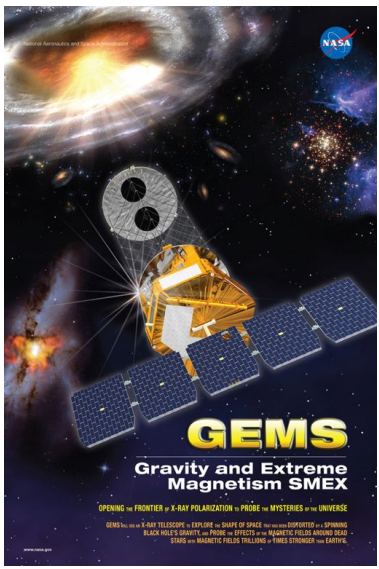




\section{Observations}

\section{Thirty Metre Telescope and Bigger}

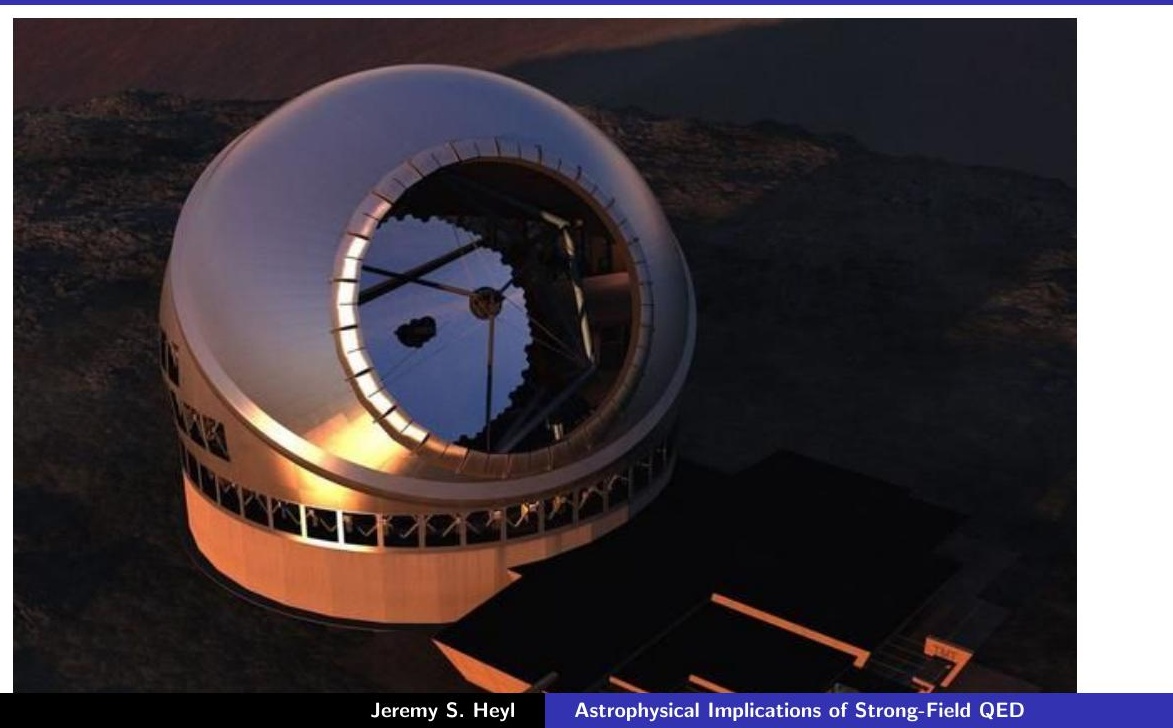




\section{Bibliography}

Adler, S. 1971, Ann. Phys., 67, 599.

Brodin, G, et al. 2007, PRL, 98, 125001.

Charonneau, J., Zhitnitsky, A. 2007, PRC, 76, 015801.

Euchner, F. et al. 2006, A\&A, 451, 671.

Gill, R., Heyl, J. 2010, MN, 407, 1926

Gill, R., Heyl, J. 2011, PRD, 84, 085001.

Goldreich, P, Julian, W. 1969, ApJ, 157, 869 (GJ).

Goldreich, P., Reisenegger, A. 1992, ApJ, 395, 250.

Heisenberg, W., Euler, W. 1936, Z. Physik, 98, 714.

Heyl, J. 2001, Phys. Rev. D, 63, 064028.

Heyl, J., Hernquist, L. 1997a, Journ. Phys. A, 30, 6485.

Heyl, J., Hernquist, L. 1997b, PRD, 55, 2449.

Heyl, J., Hernquist, L. 1997c, ApJ, 489, L67.

Heyl, J., Kulkarni, S. 1998, ApJ, 506, L61.

Heyl, J., Shaviv, N. 2000, MN, 311, 555.

Heyl, J., Shaviv, N. 2002, PRD, 66, 023002.

Heyl, J., Shaviv, N., Lloyd, D. 2003, MN, 342, 134.

Heyl, J., Shaviv, N., Lloyd, D. 2005, astro-ph/0502351.

Heyl, J. 2005a, astro-ph/0504077.

Heyl, J. 2005b, MN, 362, 777.

Heyl, J. 2007, Astrophys.Space Sci., 308, 101.

Ho, W, Lai, D. 2004, ApJ, 607, 420.

Hoffman, K., Heyl, J. S. 2009, MN, 400, 1986-1991.

Inan, U. et al. 1999, Geophysical Research Letters, 26, 3357.

Jones, P. 2006a, MN, 365, 339.

Jones, P. 2006b, MN, 371, 1327.
Jones, P. 2009, MN, 397, 1027.

Kubo, H., Nagata, R. 1983, J. Opt. Soc. Am., 73, 1719.

Lai, D., Heyl, J. 2006, PRD, 74, 123003.

Link, B, 2003, PRL, 91, 101101.

Lyutikov, M. 2006, MN, 367, 1594.

Masada, Y. et al. 2008, arXiv:0803.3818

Medin, Z., Lai, D. 2010, MN, 406, 1379.

Mereghetti, S. et al 2005, ApJ, 624, L105.

Mori, K, Heyl, J. 2007, MN, 376, 895.

Nobili, L. et al. 2008, MN, 389, 989.

Perna, R. et al. 2012, ApJ, 748, 116.

Pilia, M. et al. 2010, ApJ, 723, 707.

Pons, J. et al. 2007, PRL, 98, 071101.

Potekhin, A. 2004 et al. ApJ, 612, 1034.

Rea, N. et al. 2008, ApJ, 686, 1245.

Shaviv, N., Heyl, J., Lithwick, Y. 1999, MN, 306, 333.

Schwinger, J. 1951, PR, 82, 664.

Thirumalai, A., Heyl, J. S. 2009, PRA, 79, 12514.

Thompson, C., Duncan, R. C. 1995, MN, 275, 255.

Thompson, C., Duncan, R. C. 1996, ApJ, 473, 322.

Thompson, C., 2008, ApJ, 688, 499 \& 1258.

Wang, C., Lai, D. 2009, MN, 398, 515.

Watts, A, Reddy, S., 2007, MN, 379, L63.

Weisskopf, V. S. 1936, Kongelige Danske, 14, 6.

ApJ: Astrophysical Journal

MN: Monthly Notices of the Royal Astronomical Society 